\title{
Phase portraits of gene networks models
}

\author{
Natalia Ayupova \\ Lab. of inverse problems of \\ mathematical physics. \\ Sobolev Institute of \\ Mathematics SB RAS \\ Novosibirsk, Russia \\ ayupova@math.nsc.ru
}

\author{
Vladimir Golubyatnikov \\ Lab. of inverse problems of \\ mathematical physics. \\ Sobolev Institute of \\ Mathematics SB RAS \\ Novosibirsk, Russia \\ vpgolubyatn@gmail.com
}

\author{
Vyacheslav Gradov \\ Mechanical and \\ mathematical department \\ Novosibirsk State \\ University Novosibirsk, \\ Russia \\ v.gradov@g.nsu.ru
}

\author{
Liliya Minushkina \\ Mechanical and \\ mathematical department \\ Novosibirsk State \\ University Novosibirsk, \\ Russia \\ 1.minushkina@g.nsu.ru
}

\begin{abstract}
We investigate geometric and combinatorial structures of phase portraits of dynamical systems considered as models of functioning of some circular gene networks. Invariant 2-dimensional piecewise linear surfaces constructed here allow to give full description of phase portraits of these models and to control their trajectories.
\end{abstract}

Keywords - gene networks models, dynamical systems, phase portraits, cycles ${ }^{1}$

\section{Introduction} type

We study piecewise linear dynamical systems of the

$$
d x_{1} / d t=f_{1}\left(x_{n}\right)-k_{1} x_{1} ; d x_{j} / d t=f_{j}\left(x_{j-1}\right)-k_{j} x_{j} ;(1)
$$

as models of circular gene networks. Here $j=1,2, \ldots n ; f_{1}, f_{j}$ are monotonic step functions either increasing, or decreasing, this corresponds to positive, respectively, negative feedbacks in the gene network. Some particular cases of the systems (1) are considered in $[1,2]$. Let $\alpha_{1}, \alpha_{j}$ be discontinuity points of the functions $f_{1}, f_{j}$ and $F_{j}$ be their maximal values. We denote by $L_{j}(x)$ and $\Gamma_{j}(x)$, decreasing and increasing step functions, respectively. Our main task in these studies of phase portraits of such systems is detection of their periodic trajectories (cycles), construction of invariant surfaces and domains etc. in order to give qualitative description of trajectories of these systems. We assume that $F_{j}>k_{j}$ for all $j$, otherwise the system (1) does not have cycles, as it was shown in [3]. We say that the system (1) has the Elowitz-Leibler type ([1]) if $n=6$, and $f_{2 i-1}=L_{2 i-1}, f_{2 i}=\Gamma_{2 i}$ for $i=1,2,3$. Similarly, we say that the system (1) has the Glass-Pasternack type (see [2]) if $f_{1}=L_{1}$, and $f_{j}=\Gamma_{j}$ for $j=1,2, \ldots, n$.

\section{Discretization of Phase Portraits}

Let $Q_{n}=\left[0, F_{1}\right] \times \ldots \times\left[0, F_{n}\right]$. As in $[3,4]$, one can show that this is positively invariant domain of the system (1), and the point $A_{n}=\left(\alpha_{1}, \alpha_{2}, \ldots \alpha_{n}\right)$ is contained in $Q_{n}$. The hyperplanes $x_{j}=\alpha_{j}$ subdivide $Q_{n}$ to $2^{n}$ smaller parallelepipeds (blocks) which we enumerate by binary multi-indices $E=\left(\varepsilon_{1}, \varepsilon_{2}, \ldots, \varepsilon_{n}\right)$ as follows: $\varepsilon_{j}=0$ if $x_{j} \leq \alpha_{j}$ for all points of this block; otherwise $\varepsilon_{j}=1$. In each of these blocks trajectories of the system are described explicitly in a very simple way. We say that the block $E$ has the valence $v$ if trajectories of its points leave it through $v$ faces of this block.

\section{Results}

\section{Glass-Pasternack Systems}

It was shown in [2] that the system (1) of GlassPasternack type has a cycle $C_{1}$ which passes through 1valent blocks. For $n=4$, we construct now an invariant piecewise linear surface $S_{1}$ in the complement of union of interiors of these 1-valent blocks such that $C_{1}$ and $S_{1}$ have nontrivial link in $Q_{4}$. The surface $S_{1}$ has exactly with one vertex $A_{4}$ and eight faces. Trajectories of all points of the surface $S_{1}$ tend to $A_{4}$ as t $\rightarrow \infty$.

Elowitz-Leibler Systems

We have shown in [4] that 6-dimensional system (1) has a cycle $C_{2}$ which passes through 1-valent blocks in $Q_{6}$. Now, we construct an invariant piecewise linear surface $S_{2}$ in the union of 5-valent blocks in this invariant domain. The surface $S_{2}$ has exactly one vertex $A_{6}$. Trajectories of all points of the surface $S_{2}$ tend to $A_{6}$ as $t \rightarrow \infty$.

\section{Gene Network with Negative Feedbacks}

Consider 5-dimensional system (1) where $f_{j}=L, k_{j}=1$ for all $j=1,2, \ldots 5$. Let $F$ be maximal value of the function $L$. This system is symmetric with respect to cyclic permutation of the variables. We show that if $F>1$, and $F^{2}>5(F-1)$ then this system has two cycles. One of them passes through the union of 1-valent blocks, the second cycle is contained in the union of 3 -valent blocks. Both cycles generate invariant surfaces in phase portrait of the system.

\section{Conclusions}

Some of these invariant surfaces compose boundaries of attraction basins of stable cycles in phase portraits of dynamical systems described above

\section{ACKNOWLEDGMENT}

Supported by RFBR, grant 18-01-00057.

\section{REFERENCES}

[1] M. B. Elowitz and S. Leibler, "A synthetic oscillatory network of transcriptional regulators," Nature, vol. 403, pp. 335-338, 2000.

[2] L. Glass and J. S. Pasternack "Stable oscillations in mathematical models of biological control system," J. Theor. Biol., vol. 74, pp. 207-223, 1978

[3] V. P. Golubyatnikov and V. V. Ivanov, "Cycles in odd-dimensional models of circular gene networks," Journal of Applied and Industrial Mathematics, vol. 12, pp. 648-657, 2018

[4] V. P. Golubyatnikov and L. S. Minushkina, "Monotonicity of the Poincaré mapping in some models of circular gene networks," Journ. of Applied and Industrial Mathematics, vol. 13, pp. 472-479, 2019. 